\title{
Disruption of the Purine Nucleotide Cycle by Inhibition of Adenylosuccinate Lyase Produces Skeletal Muscle Dysfunction
}

\author{
Judith L. Swain, James J. Hines, Richard L. Sabina, \\ Olin L. Harbury, and Edward W. Holmes \\ Departments of Medicine, Physiology, and Biochemistry, \\ and the Howard Hughes Medical Institute Laboratories, Duke \\ University Medical Center, Durham, North Carolina 27710
}

bstract. Controversy exists as to whether the purine nucleotide cycle is important in normal skeletal muscle function. Patients with disruption of the cycle from a deficiency of AMP deaminase exhibit variable degrees of muscle dysfunction. An animal model was used to examine the effect of inhibition of the purine nucleotide cycle on muscle function. When the compound 5-amino-4-imidazolecarboxamide riboside (AICAriboside) is phosphorylated to the riboside monophosphate in the myocyte it is an inhibitor of adenylosuccinate lyase, one of the enzymes of the purine nucleotide cycle. AICAriboside was infused in 28 mice, and 22 mice received saline. Gastrocnemius muscle function was assessed in situ by recording isometric tension developed during stimulation. The purine nucleotide content of the muscle was measured before and after stimulation.

Disruption of the purine nucleotide cycle during muscle stimulation was evidenced by a greater accumulation of adenylosuccinate, the substrate for adenylosuccinate lyase, in the animals receiving AICAriboside $(0.60 \pm 0.10$ vs. $0.05 \pm 0.01 \mathrm{nmol} / \mu \mathrm{mol}$ total creatine, $P$ $<0.0001)$. There was also a larger accumulation of inosine monophosphate in the AICAriboside vs. salinetreated animals at end stimulation ( $73 \pm 6$ vs. $56 \pm 5$ $\mathrm{nmol} / \mu \mathrm{mol}$ total creatine, $P<0.03$ ). Inhibition of flux through the cycle was accompanied by muscle dysfunction during stimulation. Total developed tension in the AICAriboside group was $40 \%$ less than in the saline group $(3,023 \pm 1,170$ vs. $5,090 \pm 450 \mathrm{~g} \cdot \mathrm{s}, P<0.002)$. An

Dr. Swain is an Established Investigator of the American Heart Association. 1984.

Received for publication 17 April 1984 and in revised form 18 June

J. Clin. Invest.

(C) The American Society for Clinical Investigation, Inc. 0021-9738/84/10/1422/06 \$1.00

Volume 74, October 1984, 1422-1427 index of energy production can be obtained by comparing the change in total phosphagen content per unit of developed tension in the two groups. This index indicates that less high energy phosphate compounds were generated in the AICAriboside group, suggesting that interruption of the purine nucleotide cycle interfered with energy production in the muscle. We conclude from these studies that defective energy generation is one mechanism whereby disruption of the purine nucleotide cycle produces muscle dysfunction.

\section{Introduction}

The purine nucleotide cycle is a series of three reactions (Fig. 1): AMP is deaminated to inosine monophosphate (IMP) ${ }^{1}$ with liberation of ammonia; IMP and aspartate condense to form adenylosuccinate (succinyl-AMP [SAMP]) in a reaction driven by GTP hydrolysis; and SAMP is cleaved to AMP and fumarate (1). The three reactions of this cycle are catalyzed by the enzymes AMP deaminase, SAMP synthetase, and SAMP lyase. This series of reactions appears to be especially active in skeletal muscle (2). During muscle stimulation flux through the cycle increases as evidenced by the liberation of ammonia, the production of IMP, and an increase in the production of fumarate and other citric acid cycle intermediates derived from fumarate (3-6). During recovery, the ATP pool increases to resting levels with a concomitant decrease in the IMP pool to unstimulated levels $(7,8)$.

The purine nucleotide cycle is postulated to be important to normal skeletal muscle function for several reasons: $(a)$ $\mathrm{NH}_{3}$ and IMP production may regulate glycolysis (1, 9-12); (b) ammonia may buffer the hydrogen ion produced during ATP hydrolysis (13); (c) the removal of AMP may pull the myokinase reaction toward ATP production (14); (d) accumulation of IMP may be a mechanism to store purines within

1. Abbreviations used in this paper: AICAriboside, 5-amino-4-imidazolecarboxamide riboside; AICAR-TP, nucleoside triphosphate of AICAR; CP, creatine phosphate; CTP, cytidine triphosphate; GTP, guanosine triphosphate; IMP, inosine monophosphate; PRPP, phosphoribosylpyrophosphate; SAMP, succinyl-AMP; TCr, total creatine; UTP, uridine triphosphate. 




Figure 1. The purine nucleotide cycle and the metabolites of AICAriboside. The * notes the lyase, a bifunctional enzyme that catalyzes the cleavage of both succinyl-AICAR and succinyl-AMP. the cell during muscle contraction for later use in ATP synthesis during the recovery period (15); and (e) flux through the cycle generates fumarate and other citric acid cycle intermediates that enhance mitochondrial respiration $(14,16-17)$.

A deficiency of myoadenylate deaminase is associated with a more rapid onset of fatigue during maximal exercise, suggesting that the purine nucleotide cycle is important for muscle function during vigorous muscle contraction (18). However, no other genetic defects in enzymes of the purine nucleotide cycle have been reported in either humans or in animals to confirm or refute this hypothesis. In the present study we describe an animal model of a purine nucleotide cycle defect in which one of the enzymes (SAMP lyase) can be inhibited by the administration of 5-amino-4-imidazolecarboxamide riboside (AICAriboside). Prior work has shown that AICAriboside can be taken up by the myocyte and phosphorylated to the riboside monophosphate AICAR. At low intracellular concentrations of AICAR $(<70 \mu \mathrm{M})$, IMP production increases and this is associated with increased flux through the SAMP synthetase and subsequently SAMP lyase reactions, leading to increased synthesis of adenine nucleotides (19). In contrast, at high intracellular concentration of AICAR $(>190 \mu \mathrm{M})$, even though IMP production is increased adenine nucleotide production is reduced because SAMP lyase is inhibited by AICAR with an inhibition constant $\left(K_{\mathrm{i}}\right)$ of $7.6 \mu \mathrm{M}(20)$. The specificity of AICAR as an inhibitor of SAMP lyase results from a unique property of the lyase, i.e., it is a bifunctional enzyme that not only catalyzes the conversion of SAMP to AMP but also catalyzes the synthesis of AICAR from succinyl-AICAR in the de novo pathway of purine nucleotide synthesis (Fig. 1) (21). Using this animal model, we assessed physiological function in conjunction with determining the biochemical correlates of inhibition of flux through the purine nucleotide cycle.

\section{Methods}

The C57BL mice (21-31 g) used for this study were given free access to food and water until the day of the study. Animals were anesthetized with intraperitoneal pentobarbital $(65 \mathrm{mg} / \mathrm{kg})$, and a $1 \mathrm{~mm}$ o.d. catheter inserted percutaneously into the peritoneal cavity. In 28 mice an infusion of AICAriboside $(200 \mathrm{mM})$ was initiated at a rate of 17 $\mu \mathrm{l} / \mathrm{min}$. In the other 22 mice, saline (with the osmolarity adjusted to equal that of the AICAriboside solution) was infused in an identical manner. The infusion was continued during isolation of the gastrocnemius muscle in situ, and was terminated after removal of the muscle at the end of the experiment. The total volume infused during the course of the experiment averaged $0.86 \pm 0.03 \mathrm{ml}$. The left gastrocnemius muscle was carefully dissected free of surrounding tissues with its nerve and blood supply intact (22). The soleus, plantaris, and rectus femoris muscles were dissected free of the gastrocnemius muscle and removed from the preparation. The leg was secured with a steel pin through the femur and a screw clamp over the hind paw, and the limb was immersed in a $37^{\circ} \mathrm{C}$ mineral oil bath. The distal tendon of the gastrocnemius muscle was attached to a Grass FT.03 force transducer with a stainless steel rod (22). Muscle length was then adjusted to provide maximal isometric tension. Resting metabolite content was determined in six AICAriboside-treated and six saline-treated animals by freezing the muscle in situ with metal tongs precooled in liquid nitrogen. The frozen muscle was trimmed of any tissue not compressed between the tongs, and was stored in liquid nitrogen until extraction. In the 22 AICAriboside and 16 saline-treated animals undergoing muscle stimulation the transected sciatic nerve was repetitively stimulated in situ with trains of tetanizing supramaximal pulses $(8 \mathrm{~V}, 0.5$ $\mathrm{ms}, 125 \mathrm{~Hz}$ ) lasting $150 \mathrm{~ms}$ at a rate of 2 trains/s using a platinum bipolar electrode and a Grass S88 stimulator and SIU 4678 isolation unit. After $3 \mathrm{~min}$ of stimulation, the muscle was frozen in situ and stored under liquid nitrogen until extracted.

The initial tetanic tension was measured, and the total tension was determined by integrating the area under the tension-time curve during the $3 \mathrm{~min}$ of muscle stimulation. The total tension generated was used as an index of the amount of energy expended by the muscle during the three minutes of muscle stimulation.

Analysis of muscle samples. The skeletal muscle was added directly to a glass tissue grinder containing $0.4 \mathrm{ml}$ of cold $\left(4^{\circ} \mathrm{C}\right) 12 \%$ trichloroacetic acid. Extraction was performed as previously described (23) and the aqueous layer retained for analyses. Nucleotide analyses were performed using high performance liquid chromatography (20). The various peaks in the extracts were identified by comparison of retention times with known external standards and relative absorbance at 254/ $280 \mathrm{~nm}$. The mononucleotide of AICAriboside (AICAR), the de novo synthetic precursor of AICAR (succinyl-AICAR), and the nucleoside triphosphate of AICAR (AICAR-TP), were resolved using the same 
chromatographic conditions. AICAR and AICAR-TP were quantitated using the commercially available compounds (Sigma Chemical Co., St. Louis, MO) as standards. However, succinyl-AICAR is not commercially available and therefore it was synthesized and quantitated as described below. Creatine and creatine phosphate were measured utilizing paired ion-exchange chromatography as previously described (24). Nucleotide and creatine phosphate data were expressed as nanomoles per micromole of total creatine (creatine plus creatine phosphate). This method of expressing metabolite data in muscle extracts has been validated previously for both resting and stimulated muscle (24).

Synthesis of succinyl-AICAR. Succinyl-AICAR was prepared enzymatically after the method of $\mathrm{Tu}$ and Patterson (25) using yeast SAMP lyase (Sigma Chemical Co.). Enzyme ( $2 \mathrm{mg}$ protein $/ \mathrm{ml} ; 0.35$ $\mathrm{IU} / \mathrm{mg}$ protein) was solubilized in $50 \mathrm{mM}$ imidazole (pH 6.0) containing $1 \mathrm{mM}$ EDTA, and $0.9 \mathrm{IU}$ of enzyme was added to $1.5 \mathrm{mM}$ AICAR and $12.5 \mathrm{mM}$ fumarate. The mixture $(2 \mathrm{ml}$ total volume) was incubated at $37^{\circ} \mathrm{C}$ for $60 \mathrm{~min}$ and the reaction terminated by the addition of $100 \%$ trichloroacetic acid to a final concentration of $5 \%$ (vol/vol). The extract was neutralized with 2 vol of $0.5 \mathrm{M}$ tri- $\mathrm{N}$ octylamine in freon.

Succinyl-AICAR was isolated utilizing high performance liquid chromatography as described above and the concentration determined spectrophotometrically from the absorbance observed at $269 \mathrm{~nm}$ using a molar extinction coefficient of $13.3 \times 10^{3}(26)$. (Eluant collected from the same point in the salt gradient was used as the blank for these studies.) Succinyl-AICAR was also identified and its concentration determined using the modified Bratton-Marshall test for diazotable amines. This reaction yields a characteristic pink color with succinylAICAR, which has an absorbance maximum at $560 \mathrm{~nm}$. The concentration was determined using a molar extinction coefficient of 17.7 $\times 10^{3}$ at $560 \mathrm{~nm}(26)$.

One-way analysis of variance was used for comparison of metabolic data at rest vs. end-stimulation, and between AICAriboside and salinetreated groups. Comparisons of parameters of muscle function (initial tension and total developed tension) between treatment groups were made using one-way analysis of variance. All values were expressed as mean \pm SEM.

\section{Results}

Muscle function. Initial tetanic tension in the AICAribosidetreated group was $160 \pm 5 \mathrm{~g}$, and was not different from that in the saline-treated group $(162 \pm 4 \mathrm{~g})(P>0.3)$. There was no difference in initial tension between groups when the data were normalized to body weight. Fig. 2 depicts the decline in tetanic tension (expressed as percent initial tetanic tension) during the $3 \mathrm{~min}$ of sciatic nerve stimulation in the two groups. Tension declined more rapidly and to a lower minimum tension in the AICAriboside-treated group. Total developed tension during the 3 min of muscle stimulation was significantly less in the AICAriboside-treated group $(3,023 \pm 1,170 \mathrm{~g} \cdot \mathrm{s})$ than in the saline-treated group $(5,090 \pm 450 \mathrm{~g} \cdot \mathrm{s})(P<0.002)$. Thus, although initial tension was not different in the two groups, total developed tension was less in the AICAriboside-treated group.

AICAriboside and metabolites. The animals sampled at rest received $168 \pm 12 \mu \mathrm{mol}$ of AICAriboside, and those undergoing muscle stimulation received $172 \pm 6 \mu \mathrm{mol}(P>0.3)$. Neither AICAR, succinyl-AICAR, nor AICAR-TP were detectable in muscle from the animals receiving the saline

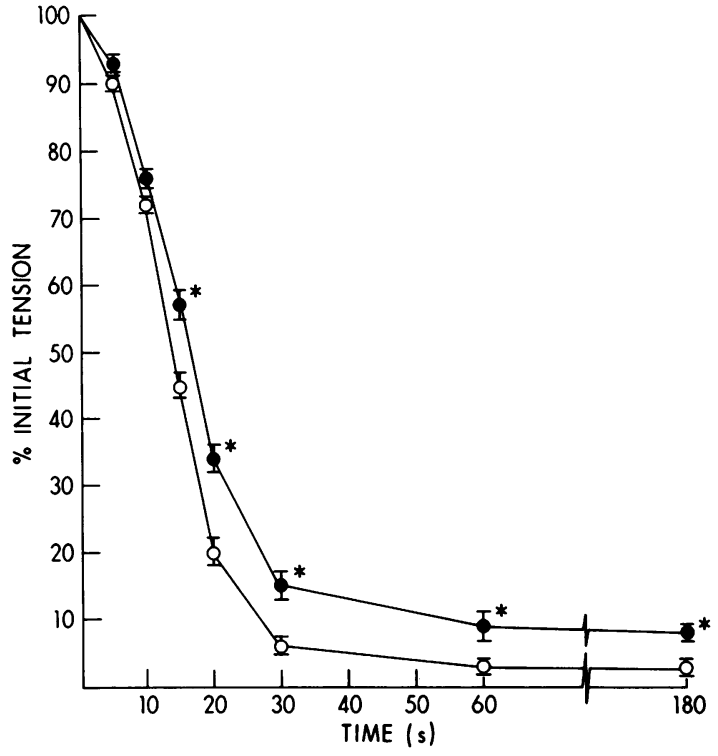

Figure 2. Percentage of initial tetanic tension during $3 \mathrm{~min}$ of stimulation of the gastrocnemius muscle with trains of tetanizing supramaximal pulses. $\bullet$, saline-treated; $O$, AICAriboside-treated. ${ }^{*} P<0.05$, AICAriboside vs. saline.

infusion, whereas all three compounds accumulated in the animals receiving AICAriboside. The content of these compounds in the muscle of the animals receiving AICAriboside is given in Table I. To express the values in terms of concentrations for comparison with enzyme kinetic data, a previously established relationship between wet weight and total creatine content of skeletal muscle was utilized (nmol/ $\mu \mathrm{mol}$ total creatine (TCr) $\div 35.8=\mu \mathrm{mol} / \mathrm{g}$ wet wt) (24). AICAR is synthesized from AICAriboside and ATP in a reaction catalyzed by adenosine kinase (Sabina, R. L., D. Patterson, and E. W. Holmes, unpublished observations). The AICAR concentration in resting muscle was estimated to be $346 \pm 38 \mu \mathrm{M}$, and that in stimulated muscle was $255 \pm 31 \mu \mathrm{M}$. These AICAR concentrations are 33-45-fold higher than the $K_{\mathrm{i}}$ values of skeletal muscle adenylosuccinate lyase for AICAR (7.6 $\mu \mathrm{M})$ (20). Succinyl-AICAR accumulated to a concentration of $25 \pm 5 \mu \mathrm{M}$

Table I. Accumulation of Metabolites of AICAriboside in Skeletal Muscle*

\begin{tabular}{lcc}
\hline & Rest $(n=6)$ & End-stimulation $(n=22)$ \\
\hline AICAR & $9.9 \pm 1.1$ & $7.3 \pm 0.9$ \\
Succinyl-AICAR & $0.71 \pm 0.15$ & $1.29 \pm 0.11$ \\
AICAR-TP & $0.30 \pm 0.07$ & $0.48 \pm 0.10$ \\
\end{tabular}

All values are expressed as nanomoles per micromole $\mathrm{TCr}$ (mean \pm SE).

* These metabolites were not detectable in muscle from the salinetreated group. 
in resting muscle and $45 \pm 4 \mu \mathrm{M}$ in stimulated muscle. Presumably, succinyl-AICAR was produced from AICAR and fumarate by reversal of the lyase reaction, since studies with cultured mammalian cells have shown this reaction to be reversible in vivo (27). AICAR-TP accumulated to a concentration of $10 \pm 2$ $\mu \mathrm{M}$ in resting muscle and $17 \pm 3 \mu \mathrm{M}$ in stimulated muscle. Presumably, AICAR-TP was synthesized from AICAR and phosphoribosylpyrophosphate (PRPP) in a reaction catalyzed by PRPP synthetase based on studies with cultured mammalian cells and purified PRPP synthetase (28).

Purine nucleotide and creatine phosphate content. The rest and end-stimulation content of the purine nucleotides AMP, ADP, ATP, IMP, and SAMP, are shown in Fig. 3 for the saline and AICAriboside-treated groups. In both groups ATP content decreased during stimulation $(P<0.0001)$, with a $23 \%$ greater decrease in ATP content in the AICAriboside-treated group $(P<0.02)$. The decrease in ATP was matched by a stoichiometric increase in IMP in both groups $(P$ $<0.0001$, rest vs. stimulation) with the increase in IMP being greater in the AICAriboside-treated group $(P<0.03)$. Total adenine nucleotide plus IMP content was similar in the two groups at rest, and did not change significantly in either group during muscle stimulation $(P>0.3)$. With stimulation SAMP increased from $10 \pm 5$ to $51 \pm 9 \mathrm{pmol} / \mu \mathrm{mol} \mathrm{TCr}$ (fivefold) in the saline group, whereas in the AICAriboside group SAMP increased from $53 \pm 11$ to $596 \pm 101 \mathrm{pmol} / \mu \mathrm{mol} \mathrm{TCr}$ (Fig. 3). The content of SAMP was greater in the AICAriboside-treated group compared with the saline group both at rest $(P<0.01)$ and at end-stimulation $(P<0.0001)$.

Creatine phosphate (CP) content decreased significantly in both the saline group $(677 \pm 16$ to $445 \pm 26 \mathrm{nmol} / \mu \mathrm{mol} \mathrm{TCr}, P$ $<0.001)$ and in the AICAriboside group $(663 \pm 28$ to $386 \pm 24$ $\mathrm{nmol} / \mu \mathrm{mol} \mathrm{TCr}, P<0.001)$ during stimulation. The mean CP content of the AICAriboside group during stimulation was less than that of the saline group, but the difference did not reach statistical significance $(P=0.10)$.

A relative index of energy (or phosphagen) production can be derived from measurements of the change in total phosphagen (ATP + CP) content of the muscle and developed

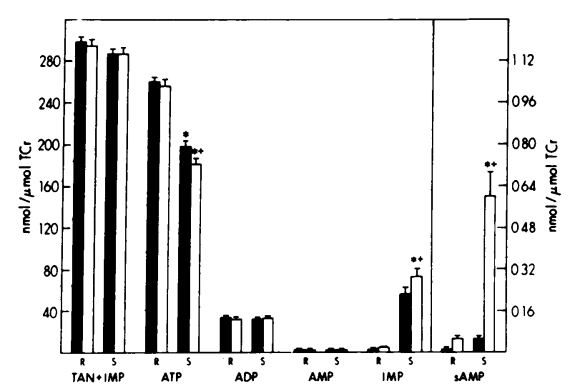

Figure 3. Content of total adenine nucleotides $($ TAN $=$ ATP + ADP + AMP) + IMP, ATP, ADP, AMP, IMP, and SAMP in skeletal muscle from saline $=(\square)$ and AICAriboside $=(\square)$ treated animals at rest $(R)$ and after stimulation (S). All values are mean \pm SEM.

${ }^{*} P<0.001$, rest vs. stimulation, ${ }^{+} P<0.05$, AICAriboside vs. saline. tension during stimulation because of the following relationship. Energy (phosphagen) utilization during muscle contraction is proportional to work performed or tension developed $(29,30)$, and the change in total phosphagen content of the muscle during stimulation reflects a balance between phosphagen (energy) consumption and phosphagen production. Thus, any difference in the change in total phosphagen content [(ATP $+\mathrm{CP})$ rest - (ATP + CP) end-stimulation] per unit of tension between the AICAriboside and saline groups reflects a difference in energy production between the two groups. The decrease in (ATP + CP) content per gram - second of tension generated was $57 \times 10^{-3} \mathrm{nmol} / \mu \mathrm{mol} \mathrm{TCr}$ in the saline group, while the decrease was approximately twofold greater $\left(116 \times 10^{-3} \mathrm{nmol} /\right.$ $\mu \mathrm{mol} \mathrm{TCr})$ in the AICAriboside group. Thus, energy production per gram $\cdot$ second of tension was $59 \times 10^{-3} \mathrm{nmol} / \mu \mathrm{mol} \mathrm{TCr}$ less in the AICAriboside group.

Other purine (guanosine triphosphate [GTP]), pyrimidine (cytidine triphosphate and uridine triphosphate [CTP, UTP]), and pyridine $\left(\mathrm{NAD}^{+}\right)$nucleotides were quantitated and no differences were detectable between the groups either at rest or during stimulation (data not shown).

\section{Discussion}

The muscle dysfunction observed in the AICAriboside-treated animals does not appear to be secondary to a nonspecific depression of muscle function since the muscle in these animals was able to generate normal initial tetanic tension. Muscle dysfunction in the AICAriboside-treated animals was characterized by a specific abnormality, i.e., a more rapid onset of fatigue and a reduction in the ability to sustain muscle contractility during maximal stimulation. These characteristics of muscle dysfunction resemble those observed in patients with a deficiency of myoadenylate deaminase, another enzyme in the purine nucleotide cycle (18). In both the animal model reported here and in prior studies with myoadenylate deaminase deficient patients, easy fatigability and diminished endurance were associated with biochemical evidence of disruption of the purine nucleotide cycle.

In the patient studies disruption of the purine nucleotide cycle results from an inherited deficiency of AMP deaminase activity, while in the present study the following observations indicate that SAMP lyase activity is reduced after administration of AICAriboside. SAMP, the substrate for the lyase reaction, accumulates to a small but significantly greater extent in resting muscle of AICAriboside-treated animals compared with saline-treated animals, and this difference is markedly exaggerated after muscle stimulation. The exaggerated accumulation of SAMP in the AICAriboside-treated animals might be explained by postulating that the greater increase in IMP concentration in these animals leads to a larger increase in SAMP synthetase activity, and this in turn results in a situation where the lyase activity becomes limiting relative to that of synthetase. If this hypothesis were correct there should be a correlation between IMP and SAMP concentrations in both the saline and AICAriboside-treated animals. As shown in Fig. 4 there is no 


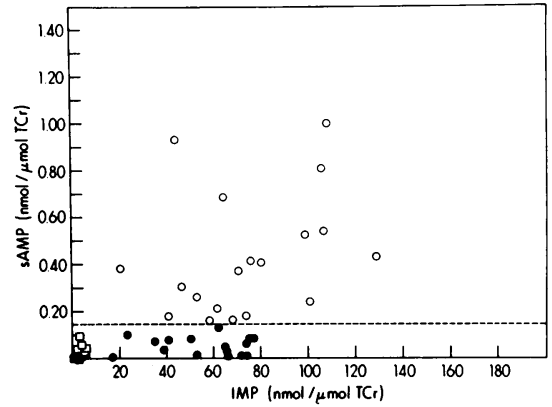

Figure 4. SAMP vs. IMP content of saline-treated animals (rest, $\mathbf{n}$; stimulation, ๑), and AICAriboside-treated animals (rest, $\square$, stimulation, o).

correlation between SAMP and IMP concentrations in the saline-treated animals, even though the IMP concentrations attained in the muscle of these animals were equal to or greater than those observed in the AICAriboside-treated group in many instances. In contrast, the SAMP concentration increased in proportion to the increase in IMP concentration in the AICAriboside-treated group. This is the expected consequence of increased SAMP synthesis in a situation where lyase activity is limiting. Thus, we conclude that the greater SAMP accumulation in the AICAriboside-treated animals is the result of inhibition of adenylosuccinate lyase rather than an inherent difference in the maximal activities of the SAMP synthetase and SAMP lyase enzymes in skeletal muscle. This conclusion is supported by the demonstration that maximal activities of the synthetase and lyase are approximately equal in vivo (31) and that situations that lead to marked increases in flux through the purine nucleotide cycle in vitro do not lead to SAMP accumulation to any appreciable extent (32).

Inhibition of adenylosuccinate lyase activity in the muscle of AICAriboside-treated animals most likely results from the accumulation of AICAR. AICAR is a competitive inhibitor of the lyase (20), and the estimated intracellular concentration of AICAR attained in this study exceeds the $K_{\mathrm{i}}$ of the lyase by 40-fold. AICAR in the concentrations attained in this study is not an inhibitor of SAMP synthetase or AMP deaminase (unpublished observations). The relative specificity of this inhibitor may be attributed to a unique property of SAMP lyase. SAMP lyase is a bifunctional enzyme (21), and AICAR is a product of the reaction catalyzed by the lyase reaction in the de novo pathway (see Fig. 1). (When AICAriboside is administered in significantly lower concentrations than used in this study, AICAR is rapidly metabolized to IMP and flux through the SAMP synthetase and lyase reactions increases. At low concentrations AICAriboside has been used to effectively increase adenine nucleotide synthesis in vivo in cardiac muscle [19].)

As pointed out earlier, disruption of the purine nucleotide cycle as a result of AICAriboside administration, or as a result of myoadenylate deaminase deficiency, leads to a specific type of defect in muscle function, i.e., easy fatigability and reduced endurance for maximal contractility. Results of this study suggest at least one mechanism for this type of defect in muscle performance. If the assumptions underlying the estimation of energy production are valid, disruption of the purine nucleotide cycle secondary to AICAriboside administration results in a significant decrease in energy production. Similar considerations in myoadenylate deaminase deficient patients have led to a similar conclusion (18). A defect in energy production secondary to decreased flux through the purine nucleotide cycle could be explained by a reduction in fumarate production. Argon and Lowenstein (3) have documented that the purine nucleotide cycle is the major source of the increase in citric acid cycle intermediates in skeletal muscle during vigorous muscle contraction. This anaplerotic role of the purine nucleotide cycle in producing citric acid cycle intermediates has been shown to have a catalytic effect of enhancing mitochondrial respiration (17). AICAriboside administration could result in a decrease in fumarate availability for mitochondria in two ways; by inhibition of SAMP lyase, and by reversal of the succinyl-AICAR lyase reaction, resulting in the consumption of fumarate. Both of these predictions are supported by the finding of SAMP and succinyl-AICAR accumulation in the skeletal muscle of these animals.

\section{Acknowledgments}

The authors wish to acknowledge the technical assistance of Margaret C. Evans and the secretarial assistance of Carolyn S. Mills.

This work was supported by grants HL 26831 and AM 12413 from the National Institutes of Health.

\section{References}

1. Lowenstein, J. M., and M. N. Goodman. 1978. The purine nucleotide cycle in skeletal muscle. Fed. Proc. 37:2308-2312.

2. Purzycka, J. 1962. AMP and adenosine aminohydrolases in rat tissues. Acta Biochim. Pol. 9:83-93.

3. Aragon, J. J., and J. M. Lowenstein. 1980. The purine-nucleotide cycle. Comparison of the levels of citric acid cycle intermediates with the operation of the purine nucleotide cycle in rat skeletal muscle during exercise and recovery from exercise. Eur. J. Biochem. 110:371377.

4. Goodman, M. N., and J. M. Lowenstein. 1977. The purine nucleotide cycle. Studies of ammonia production by skeletal muscle in situ and in perfused preparations. J. Biol. Chem. 252:5054-5060.

5. Tornheim, K., and J. M. Lowenstein. 1972. The purine nucleotide cycle. The production of ammonia from aspartate by extracts of rat skeletal muscle. J. Biol. Chem. 217:162-169.

6. Meyer, R. A., G. A. Dudley, and R. L. Terjung. 1980. Ammonia and IMP in different skeletal muscle fibers after exercise in rats. $J$. Appl. Physiol. 49:1037-1041.

7. Meyer, R. A., and R. L. Terjung. 1979. Differences in ammonia and adenylate metabolism in contracting fast and slow muscle. Am. $J$. Physiol. 6:C111-Ci18.

8. Meyer, R. A., and R. L. Terjung. 1980. AMP deamination and IMP reamination in working skeletal muscle. Am. J. Physiol. 239:C32C38.

9. Tornheim, K., and J. M. Lowenstein. 1973. The purine nucleotide 
cycle. III. Oscillations in metabolite concentrations during the operation of the cycle in muscle extracts. J. Biol. Chem. 248:2670-2677.

10. Tornheim, K., and J. M. Lowenstein. 1974. The purine nucleotide cycle. IV. Interactions with oscillations of the glycolytic pathway in muscle extracts. J. Biol. Chem. 249:3241-3247.

11. Tornheim, K., and J. M. Lowenstein. 1975. The purine nucleotide cycle. Control of phosphofructokinase and glycolytic oscillations in muscle extracts. J. Biol. Chem. 250:6304-6314.

12. Aragon, J. J., K. Tornheim, and J. M. Lowenstein. 1980. On a possible role of IMP in the regulation of phosphorylase activity in skeletal muscle. FEBS (Fed. Eur. Biochem. Soc.) Lett. 117:K56-K64.

13. Hochachka, P. W., and T. P. Mommsen. 1983. Protons and anaerobiosis. Science (Wash. DC). 219:1391-1397.

14. Lowenstein, J. M. 1972. Ammonia production in muscle and other tissues. The purine nucleotide cycle. Physiol. Rev. 52:384-414.

15. Sabina, R. L., J. L. Swain, B. M. Patten, T. Ashizawa, W. E. O'Brien, and E. W. Holmes. 1980. Disruption of the purine nucleotide cycle. A potential explanation for muscle dysfunction in myoadenylate deaminase deficiency. J. Clin. Invest. 66:1419-1423.

16. Aragon, J. J., K. Tornheim, M. N. Goodman, and J. M. Lowenstein. 1981. Replenishment of citric acid cycle intermediates by the purine nucleotide cycle in rat skeletal muscle. In Current Topics Cellular Regulation. Academic Press, Inc., New York. 18:131-149.

17. Scislowski, P. W. D., Z. Aleksandrowicz, and J. Swierczynski. 1982. Purine nucleotide cycle as a possible anaplerotic process in rat skeletal muscle. 1982. Experientia. 38:1035-1037.

18. Sabina, R. L., J. L. Swain, C. W. Olanow, W. G. Bradley, W. N. Fishbein, S. DiMauro, and E. W. Holmes. 1984. Myoadenylate deaminase deficiency. Functional and metabolic abnormalities associated with disruption of the purine nucleotide cycle. J. Clin. Invest. 73:720730 .

19. Swain, J. L., J. J. Hines, R. L. Sabina, and E. W. Holmes. 1982. Accelerated repletion of ATP and GTP pools in post-ischemic canine myocardium using a precursor of purine de novo synthesis. Circ. Res. 51:102-105.

20. Sabina, R. L., K. H. Kernstine, R. L. Boyd, E. W. Holmes, and J. L. Swain. 1982. Metabolism of 5-amino-4-imidazolecarboxamide ribonucleoside in cardiac and skeletal muscle. Effects on purine nucleotide synthesis. J. Biol. Chem. 257:10178-10183.

21. Holmes, E. W. 1978. Regulation of purine biosynthesis de novo. In Uric Acid. W. N. Kelley and I. M. Weiner, editors. SpringerVerlag, New York. 21-42.

22. Hettleman, B. D., R. L. Sabina, M. K. Drezner, E. W. Holmes, and J. L. Swain. 1983. Defective adenosine triphosphate synthesis. An explanation for skeletal muscle dysfunction in phosphate-deficient mice. J. Clin. Invest. 72:582-589.

23. Swain, J. L., R. L. Sabina, P. A. McHale, J. C. Greenfield, and E. W. Holmes. 1982. Prolonged myocardial nucleotide depletion after brief ischemia in the open-chest dog. Am. J. Physiol. 242:H818-H826.

24. Sabina, R. L., J. L. Swain, J. J. Hines, and E. W. Holmes. 1983. A comparison of methods for quantitation of metabolites in skeletal muscle. J. Appl. Physiol. 55:624-627.

25. Tu, A. S., and D. Patterson. 1977. Biochemical genetics of Chinese hamster cell mutants with deviant purine metabolism. VI. Enzymatic studies of two mutants unable to convert inosinic acid to adenylic acid. Biochem. Genetics. 15:195-210.

26. Lukens, L. N., and J. M. Buchanan. 1959. Biosynthesis of the purines. XXIII. The enzymatic synthesis of $N$-(5-amino-1-ribosyl-4imidazolylcarbonyl)-L-aspartic acid 5'-phosphate. J. Biol. Chem. 234:1791-1798.

27. Sabina, R. L., D. Patterson, and E. W. Holmes. 1983. Metabolic fates of 5-amino-4-imidazolecarboxamide riboside in eukaryotic cells. Fed. Proc. 42:2209. (Abstr.)

28. Sabina, R. L., M. A. Becker, and E. W. Holmes. 1984. The enzymatic synthesis of 5-amino-4-imidazolecarboxamide riboside triphosphate (ZTP). Science (Wash. DC). 223:1193-1195.

29. Holloszy, J. O., W. W. Winder, R. H. Fitts, M. J. Rennie, R. C. Hickson, and R. K. Conlee. 1976. Energy production during exercise. In Third International Symposium on Biochemistry of Exercise. F. Landry and W. A. R. Orvan, editors. Symposia Specialists, Inc., Miami, FL. 61-74.

30. Infante, A. A., and R. E. Davies. 1965. The effect of 2,4 dinitrofluorobenzene on the activity of striated muscle. J. Biol. Chem. 210:3996-4001.

31. Scholtz, V., and J. M. Lowenstein. 1976. Purine nucleotide cycle. Evidence for the occurrence of the cycle in brain. J. Biol. Chem. 251:485-492.

32. Manfredi, J. P., and E. W. Holmes. 1983. Control of the purine nucleotide cycle (PNC): Effects of energy state and concentration of cycle intermediates. Fed. Proc. 42:326. (Abstr.) 